\title{
Cosmic Rays Energy Spectrum from PeV to EeV energies measured by the TALE detector
}

\author{
Tareq AbuZayyad for the Telescope Array Collaboration ${ }^{* \dagger}$ \\ University of Utah \\ E-mail: tareq@cosmic.utah.edu
}

\begin{abstract}
We report on a cosmic ray energy spectrum measurement by the Telescope Array Low-Energy extension (TALE) air fluorescence detector FD). This spectrum exhibits two features, the second knee in the lower part of the $10^{17} \mathrm{eV}$ decade, and a hardening in the lower part of the $10^{16} \mathrm{eV}$ decade. The energies of these features, for the first time, are tied to the energy scale of fluorescence detectors of ultra-high energy cosmic rays. The TALE FD is sensitive to both fluorescence light and to the Cherenkov light produced by shower particles. Since Cherenkov light is very bright, we measure the energy spectrum from a low energy of $4 \mathrm{PeV}$ to an energy greater than $100 \mathrm{PeV}$ in Cherenkov light. Above $100 \mathrm{PeV}$ the detection of cosmic rays by their air fluorescence emission becomes more efficient (larger air volume) and it allows for the extension of the measurement to energies greater than a few EeV.
\end{abstract}

35th International Cosmic Ray Conference - ICRC2017-

10-20 July, 2017

Bexco, Busan, Korea

\footnotetext{
* Speaker.

${ }^{\dagger}$ full author list and acknoledgments at: http://www.telescopearray.org/images/papers/ICRC2017-authorlist.pdf
} 


\section{Introduction}

The Telescope Array (TA) is the largest detector in the northern hemisphere for studying ultrahigh energy cosmic rays. Its is the successor to the AKeno Giant Air Shower Array (AGASA) and the High Resolution Fly's Eye (HiRes) experiments [1, 2], designed with the goal of improving on both. TA is composed of three fluorescence detectors (FD's) [3, 4] and a large array of surface detectors [5] located in Millard County, Utah. The surface detector array is made up of 507 scintillation counters with $1.2 \mathrm{~km}$ spacing on a square grid. The three fluorescence detectors each covers elevation angles between $3-31^{\circ}$, and a range of azimuthal angles of about 110 overlooking the SD array. The arrangement of the TA detectors are shown in the left panel of Figure 1.

Since 2011, TA has been constructing a low energy extension (TALE) [6]. TALE has a fluorescence detector of ten telescopes at the TA Middle Drum (MD) site. They observe between $31-59^{\circ}$ in azimuth, above the field of view of the original TA FD telescopes at the MD site. The arrangement of the ten TALE telescopes are shown in the right panel of Figure 1. In addition, TALE will also operate an in-fill array of 111 surface detectors, arranged with varying detector spacing under the field of view of the TALE FD. As of summer 2017, the deployment of the TALE SD array is about half done. The remaining array will be completed during the fall of 2017 . The fluorescence detector was completed in the fall of 2013, and has been taking data in monocular mode since that time. The motivation for TALE is to extend the energy reach of TA to below $10^{16}$ $\mathrm{eV}$. In particular the transition from cosmic rays of galactic origin to those of extra-galactic origin is suspected to occur between $10^{17}$ to $10^{18} \mathrm{eV}$. TALE will provide both FD and SD measurements of the energy spectrum, as well as $x_{\max }$-based composition study in this energy range. We expect to see correlated changes in spectral index and in the composition mix.
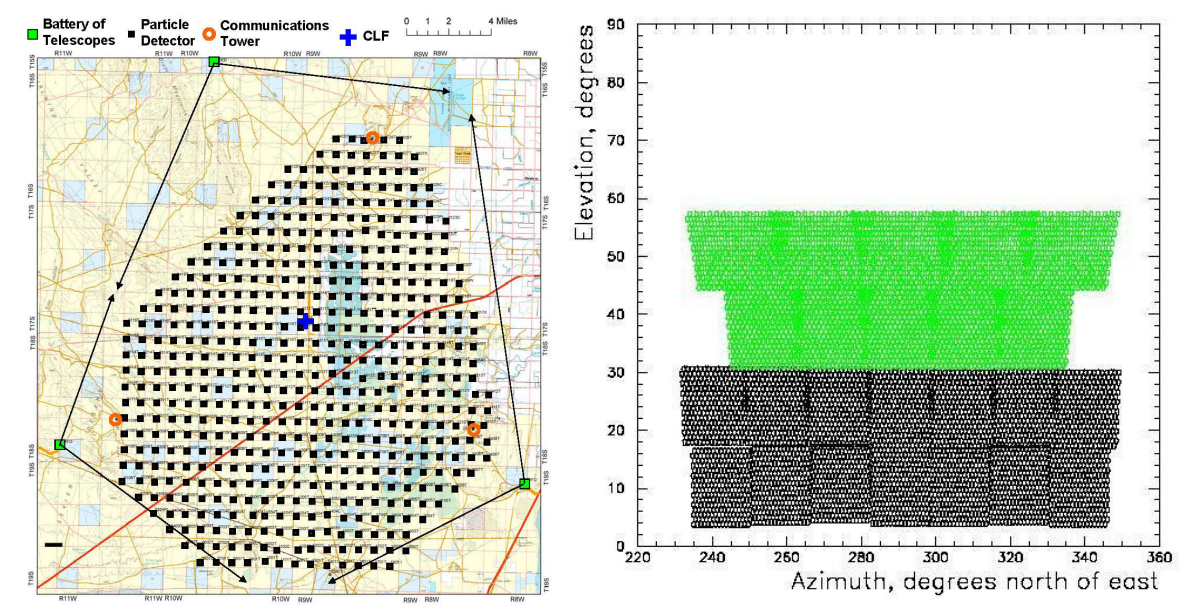

Figure 1: Left: Map of the Telescope Array surface detector and the three fluorescence detectors overlooking the array. The MD site is at the green square at the top of the map. Right, schematic of TALE/MD mirrors showing elevation coverage.

\section{Monocular Event Reconstruction}

The analysis of the monocular data exploits the capabilities of the TALE FD as fluorescence 
detectors at energies above $10^{17} \mathrm{eV}$, and gradually transitioning to imaging air Cherenkov telescopes (IACT) at energies below $10^{16.5} \mathrm{eV}$. Taking advantage of the Cherenkov light, we are able to measure the energy spectrum down to $10^{15.6} \mathrm{eV}$.

TALE FD events have short angular track-lengths. Monocular reconstruction therefore uses a combined geometry/profile reconstruction procedure, referred to as the Profile Constrained Geometry Fit (PCGF) [7]. This technique was originally developed for the analysis of monocular HiRes-I data, and was successfully used to make the first observation of the Greisen-Zatsepin-K'uzmin (GZK) Cut-off [8].

When applied to the Cherenkov-dominated events, we found that the profile constraint method gives very precise geometrical reconstruction, due primarily to the sharply peaked (in the forward direction) nature of the Cherenkov light. As will be seen in section 4, the resulting resolutions are comparable to that for hybrid or a stereo observation of fluorescence-dominated events at higher energies.

\section{Simulation}

A detailed simulation of the detector optics and electronics response to fluorescence and Cherenkov light is used to calculate the detector aperture, and to study the resolution of the event reconstruction. The shower development curves and the lateral spread of the shower particles are described using parametrization based on fits to output from CORSIKA [14]. In particular we make use of the IACT package [9] within CORSIKA to do a direct simulation of the Cherenkov light production. The latter option makes no parametrization of the lateral angular distribution of the Cherenkov signal, and incorporates shower-to-shower fluctuations in the light production of assuming some average behavior.

\section{Reconstruction Performance}

We use the CORSIKA/IACT simulations to quantify the reconstruction resolution. This represents a more stringent test than would be had we used parametrized showers; since the same parametrization are used in the reconstruction. Also, this serves as a test of the magnitude of the error introduced by ignoring the geomagnetic field in the reconstruction. An interface to our detector simulation program was developed to allow it to read in the photons arriving at the site as calculated by the external simulation, and was used to connect the two simulation packages. We simulated and reconstructed a set of mono-energetic iron and proton showers $(E=2,3,6$, and 10 $\mathrm{PeV}$ ) produced by the CORSIKA/IACT. We then simulated the same events (same geometry, fitted CORSIKA profiles) using the standard detector MC program with parameterized showers. We compared the reconstruction of both types of simulations and verified that the reconstruction performance is the same for both, i.e. similar resolution and bias for track geometry, shower energy and $x_{\max }$. With this knowledge we use parameterized showers for a more complete study of the reconstruction performance.

Figure 2 shows the energy reconstruction results. A mixed primary composition was thrown with a power law distribution in energies (spectral index -2.92). The histograms are summed over 
energy for three sets of simulations starting at different energies but all going up to an energy of about $\log (E[E e V])=18.5$. The starting energies are $\log (E[E e V])=15.3,16.2$, and 16.8.
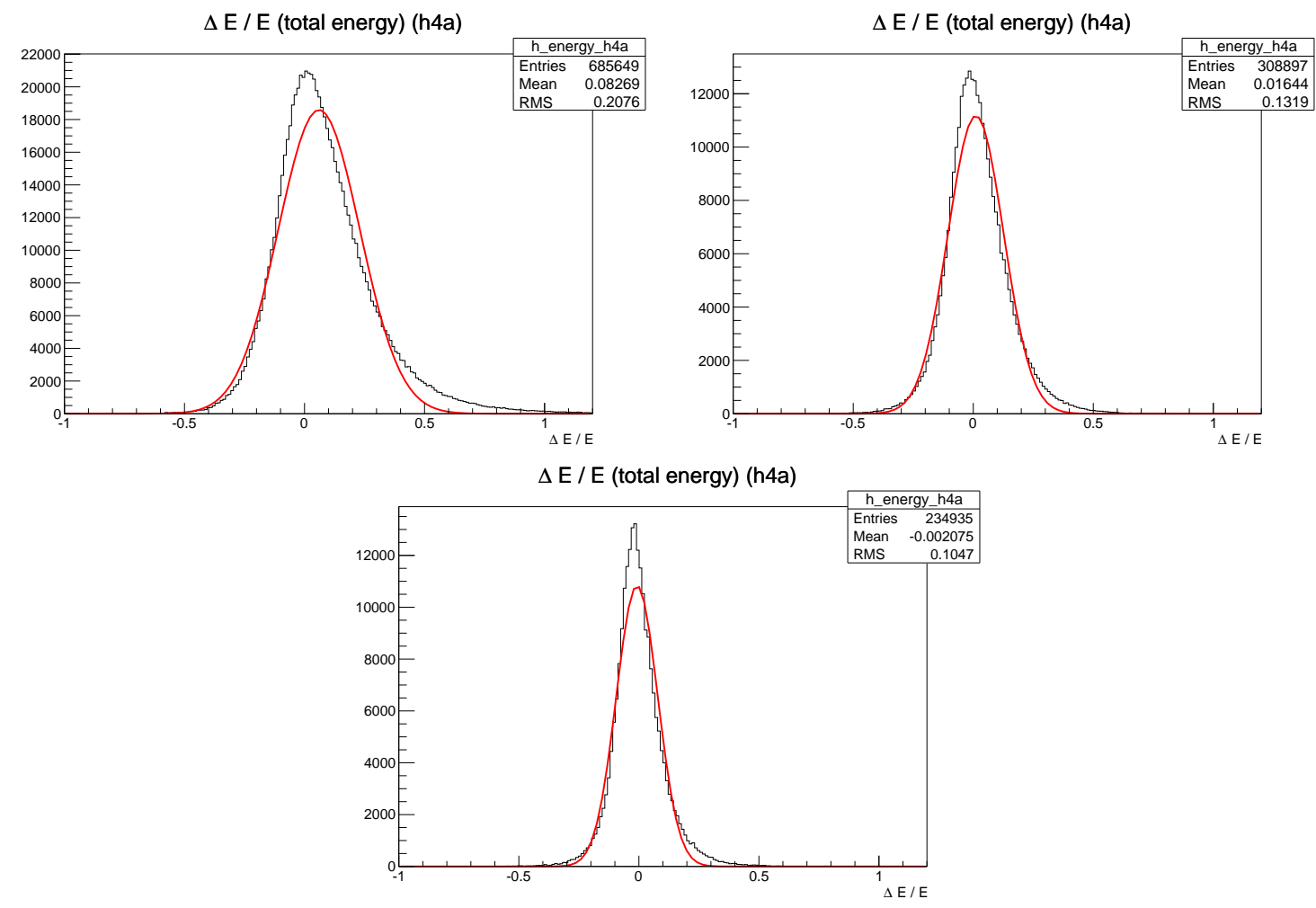

Figure 2: Shower energy resolution after all quality cuts are applied. The MC was thrown using a mixed composition with a power law in energy, spectral index $=-2.92$. The distributions are cumulative with starting energies of $\log (E[E e V])=15.3$ (top left), 16.2 (top right), and 16.8 (bottom).

\section{Aperture Calculation}

The detector aperture is calculated using different composition assumptions. The first is the H4a model [10]. In addition to H4a, we also examined the effect of changing the model to the "Global Fit" model described in [11]. Finally, we attempted to derive from the TALE data itself, a "primary mixture" which would produce $x_{\max }$ distributions which match the observed distributions. This last assumption represents a first attempt at inferring the composition and should be understood to be a very preliminary result; a work in progress.

Simulated showers are reconstructed using the same procedure applied to real data, and event selection is done in the same way as well. A missing energy correction is applied to the reconstructed data and MC showers based on the same composition assumption made, with the correction for each primary type and energy is estimated from the CONEX [13] generated showers (cross checked against CORSIKA predictions). CONEX version 4.36 with QGSJet II-3 was used. A separate MC set using QGSJet II-4 (CONEX version 4.37) was produced as well. The measured spectrum using data from the operation period of 06/2014 to 03/2016 is shown in figure 3. This result is based on an analysis using QGSJet II-3. In addition, while a small correction to the H4a 
model, we also use a composition assumption based on the HiRes-MIA measurement above $10^{17}$ $\mathrm{eV}[12]$.

\section{Results and Discussion}

The measured flux using the three different thrown primary mixtures is shown in figure 3 . As can be immediately seen from the figure, the absolute magnitude of the flux depends on the primary mixture. The TALE $x_{\max }$ distributions favor a light composition, which means a bigger aperture and a smaller total event energy (smaller missing energy correction). The combined effect of these two factors is a lower flux estimate.

We do note however that the qualitative features of the spectrum are the same. Namely the dip at about $10^{16.3} \mathrm{eV}$ and the "second knee" at $10^{17} \mathrm{eV}$ can be clearly seen in all three cases.

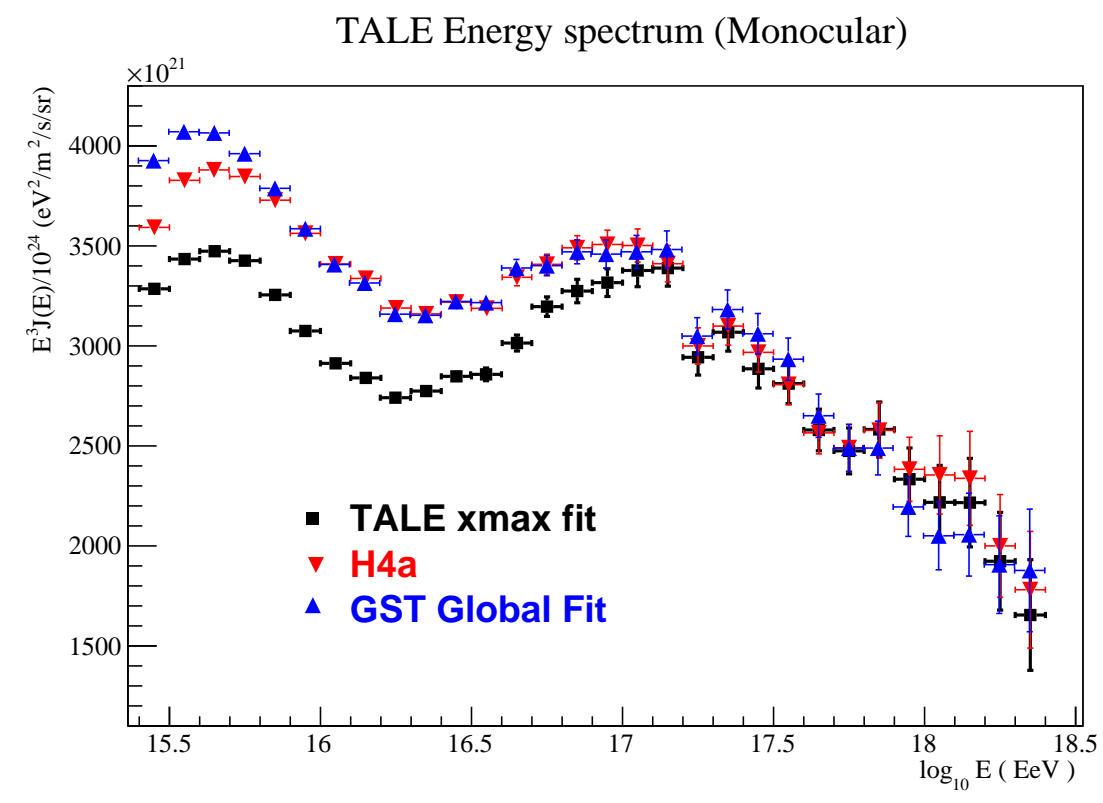

Figure 3: TALE Cosmic rays energy spectrum measured with TALE. The result is based on a QGSJet II-3 hadronic model assumption. A mixed primary composition given by the $\mathrm{H} 4 \mathrm{a}$, and "global fit" models, as well as a TALE derived mix was used in the calculations. 


\section{References}

[1] Teshima, M. and Ohoka, H. and Matsubara, Y. and Hara, T. and Hatano, Y. et al., Expanded Array for Giant Air Shower Observation at Akeno, Nucl.Instrum.Meth. A247, 399 (1986)

[2] Sokolsky, P., Final Results from the High Resolution Fly's Eye (HiRes) Experiment, Nucl.Phys.Proc.Suppl. 212-213, 74-78 (2011)

[3] Abu-Zayyad, T. and Aida, R. and Allen, M. and Anderson, R. and Azuma, R. et al., The Energy Spectrum of Telescope Array's Middle Drum Detector and the Direct Comparison to the High Resolution Fly's Eye Experiment, Astropart.Phys. 39-40, 109-119 (2012)

[4] Tokuno, H. and Tameda, Y. and Takeda, M. and Kadota, K. and Ikeda, D. et al., New air fluorescence detectors employed in the Telescope Array experiment, Nucl.Instrum.Meth. A676, 54-65 (2012)

[5] Abu-Zayyad T. et al., The Surface Detector Array of the Telescope Array Experiment, Nucl.Instrum.Meth. A689, 87-97 (2012)

[6] G.B. Thomson et al., The Telescope Array Low Energy Extension (TALE), in proceedings of International Cosmic Ray Conference 3, 337-339 (2011)

[7] Abu-Zayyad, T., The Energy Spectrum of Ultra High Energy Cosmic Rays, PhD thesis, University of Utah (2000)

[8] Abbasi, R.U. et al., First Observation of the Greisen-Zatsepin-Kuzmin suppression, Phys.Rev.Lett. 100, 101101 (2008)

[9] Bernlohr, Konrad, Simulation of Imaging Atmospheric Cherenkov Telescopes with CORSIKA and sim_telarray, Astropart.Phys. 30, 149-158 (2008)

[10] Gaisser, T.K., Spectrum of cosmic-ray nucleons, kaon production, and the atmospheric muon charge ratio, Astropart.Phys. 35 801-806 (2012)

[11] Gaisser, Thomas K. and Stanev, Todor and Tilav, Serap, Cosmic Ray Energy Spectrum from Measurements of Air Showers, Front. Phys.(Beijing) 8 748-758 (2013)

[12] Abu-Zayyad, T. et al. [HiRes-MIA Collaboration], Measurement of the cosmic ray energy spectrum and composition from $10^{17} \mathrm{eV}$ to $10^{18.3} \mathrm{eV}$ using a hybrid fluorescence technique, Astrophys. J. 557, 686-699 (2001)

[13] Bergmann, T. et al., One-dimensional Hybrid Approach to Extensive Air Shower Simulation, Astropart.Phys. 26, 420-432 (2007)

[14] Heck, D. et al., CORSIKA: A Monte Carlo code to simulate extensive air showers, FZKA-6019 (1998)

[15] Kamata, K., and Nishimura, J., Prog. Theor. Phys. Suppl. 693 (1958)

[16] Greisen, K., Prog. Cosm. Ray Phys. 31 (1956) 